\title{
Introduction to the special issue on NorCAS 2017, the 3rd Nordic circuits and systems conference
}

\section{J. Jacob Wikner ${ }^{1}$}

Published online: 15 February 2019

(C) Springer Science+Business Media, LLC, part of Springer Nature 2019

This Special Issue includes selected papers from the 3rd NorCAS conference held in Linköping, Sweden, November 2017. The NorCAS conference is the result of a merger between the former International Symposium on Systemson-Chip and the Norchip conference which has been held annually since 1983. A Special Issue from this conference has been published by Springer since the 1992 Norchip conference in Helsinki, Finland. The special issues are not only an excerpt of selected papers from the conference, but also good snapshots of research trends throughout the years.

The NorCAS conference covers research topics spanning digital systems to analog and mixed-mode integrated circuits. This special issue has selected seven papers among those 20-odd papers focusing on analog and RF circuits. The selected papers cover topics in energy harvesting, sensor interfaces and RF circuits.

1. "Self-powered micro-watt level piezoelectric energy harvesting system with wide input voltage range" is a work supported by a Horizon 2020 program and describes a $180-\mathrm{nm}$ integrated circuit with rectifiers and DC-DC converters to be used together with vibrational harvesters.

2. “A sub-nW Neuromorphic Receptor for Wide-Range Temporal Patterns of Post-Synaptic Responses in $65 \mathrm{~nm}$ CMOS" deals with the design of synaptic circuit modelling neural receptors.

3. "Design and Analysis of a Three-Dimensional Millimeter-wave Frequency-shift based CMOS Biosensor using Vertically Stacked Spiral Inductors in LC Oscillators" deals with the design of circuitry that can detect frequency shifts in LC oscillators tuned at

J. Jacob Wikner jacob.wikner@liu.se

1 Department of Electrical Engineering, Linköping University, 58183 Linköping, Sweden
$60 \mathrm{GHz}$. The frequency shift is a function of the biomolecular structure of the substance under test.

4. "A stacked transistor approach to millimeter wave SiGe power amplifiers" describes a design methodology for PAs and the authors present results from two implementations operating in the $\mathrm{Ka}$ and $\mathrm{W}$ bands, respectively.

5. "A Four Channel Phased Array Transmitter using an Active RF Phase Shifter for 5G Wireless Systems" describes the implementation of an RF transmitter including measurement results. The design is implemented in a 45-nm CMOS process.

6. "A Fully Integrated $4 \times 2$ Element CMOS RF Phased Array Receiver for $5 \mathrm{G}$ " describes the implementation and measurement result of a 45-nm integrated CMOS $\mathrm{RF}$ receiver.

7. “A 71-76 GHz wideband receiver front-end for phased array applications in $0.13 \mu \mathrm{m}$ SiGe BiCMOS technology", the last paper in the special issue, also deals with phased-array designs and shows the implementation and measurement results of a receiver with improvements of e.g. the LNA in the signal chain.

The papers presented in this special issue have been subject to peer reviews and each paper has undergone at least one revision as a consequence of the comments from reviewers. I, together with the conference organizers, would like to thank the reviewers and the Editor-in-Chief Mohammed Ismail for encouraging us to prepare this Special Issue and the Editorial Office at Springer for the efficient management of the review procedure for this Special Issue.

J. Jacob Wikner is a Reader ("Docent") in electronics systems at Linköping University (LiU). He received his M.Sc. in computer science and engineering from LiU, Sweden, in 1996. He earned his Ph.D. in 2001 on the topic "Studies on CMOS Digital-to-Analog Converters" from the electronics systems group, at the Department of Electrical Engineering (ISY) at LiU. During 2001, he spent time as a visiting post-doc at the Imperial College, London, UK. Between 1999 and 2002, he worked as a research engineer at the microelectronics research center (MERC) at Ericsson Microelectronics. Between 2003 
and 2005, he was with Infineon Technologies as a senior analog design engineer. In 2005, he joined Sicon Semiconductor in Linköping, Sweden, as a senior design engineer and chip architect. His field of work has spanned over image sensors, transceivers for wired and wireless standards, video digitizers and x-ray image sensors. Since August 2009, he is with the integrated circuits and systems group (ICS) at Linköping University as an associate professor (Bitr. Prof.). His research include biologically inspired architectures (BIAs), high-speed A/D and D/A converters, and general analog and mixed-signal design topics. The research projects range from lowvoltage and low-power analog interfaces to environment measurements through in-vivo blood-flow measurements and body-coupled communication in the http://BioComLab.se project. He has supervised ten Ph.D. students, $>100$ master student's projects, and is responsible for and lecturer in several courses in mixed-signal inte- grated circuit design as well as basic electronic and electrical circuits courses. He holds eight patents, has published some 70 journal and conference papers, and co-authored the book CMOS Data Converters for Telecommunication. He is a co-founder of one research-based company, CogniCatus $\mathrm{AB}$, and a design-services company, AnaCatum Design $\mathrm{AB}$ (now acquired by Fingerprint Cards $\mathrm{AB}$ ), both in Linköping.

Publisher's Note Springer Nature remains neutral with regard to jurisdictional claims in published maps and institutional affiliations. 